\title{
Radiolabelled peptides in diagnosis and therapy: an introduction
}

\author{
Adil AL-Nahhas $\cdot$ Stefano Fanti
}

Published online: 3 March 2012

(C) Springer-Verlag 2012

The emergence of peptide receptor radionuclide imaging (PRRI) is highly regarded as a landmark in the diagnosis of tumours and other diseases through interaction with cell membrane receptors. The small size and low molecular weight of peptides, compared to proteins and antibodies, facilitate rapid access to target tissue and similarly, fast clearance from nontarget tissue. The resultant high tumour to background ratio provides a unique opportunity to map the distribution and assess the severity of a variety of tumours by planar and single photon emission computed tomography (SPECT) of ${ }^{111} \mathrm{In}$ and ${ }^{99 \mathrm{~m}} \mathrm{Tc}$ labelled peptides. The emergence of radiolabelled peptides for use with PET/ CT such as ${ }^{68} \mathrm{Ga},{ }^{18} \mathrm{~F}$ and ${ }^{64} \mathrm{Cu}$, and the use of new receptor binding ligands such as the various DOTA compounds, have revolutionized PRRI and improved its diagnostic power beyond expectation. The inevitable move to utilize this novel technique to deliver peptide receptor radionuclide therapy (PRRT) followed swiftly by replacing diagnostic with therapeutic radionuclides such as ${ }^{90} \mathrm{Y}$ and ${ }^{177} \mathrm{Lu}$. The main use of PRRI and PRRT remains at present largely restricted to neuroendocrine tumours (NET) expressing somatostatin receptors, but efforts to export these novel

\footnotetext{
A. AL-Nahhas

Department of Nuclear Medicine, Imperial College NHS Trust, London, UK

S. Fanti

Department of Nuclear Medicine,

University Hospital S. Orsola-Malpighi,

Bologna, Italy

\author{
A. AL-Nahhas $(\bowtie)$ \\ Department of Nuclear Medicine, Hammersmith Hospital, \\ Du Cane Road, \\ London W12 0HS, UK \\ e-mail: adil.al-nahhas@imperial.nhs.uk
}

techniques to other tumours expressing the same or different receptors are in progress.

The rapid proliferation of PRRI and PRRT over a relatively short period of time is remarkable, as is the expanding research aiming at using new peptides that target different receptors in different types of tumours. All these factors combine to make it mandatory to dedicate a supplement of the EJNMMI to this subject. This supplementary issue has been complied by a group of experts in the field, some of whom are pioneers and leaders in the European and international platform. In addition to providing a historical background, they aim at highlighting the state-of-the-art as it stands now by comparing their experience with that of their contemporaries in an effort to reach a consensus. Other contributors offer a glimpse of the available research and what the future holds in this exciting arena.

A good starting point in such a comprehensive review is to understand the rationale for the use of radiolabelled peptides, and Koopmans and Glaudemans [1] explore the development of peptides and the criteria required for imaging purposes with an overview of existing radiolabelled peptides in oncological and other diseases. Fani and Maecke of the Basel group [2] look at the radiopharmaceutical development of radiolabelled peptides with its multiple steps of identification of a target, synthesis of a peptide, radiolabelling, in vitro characterization and evaluation followed by use in clinical studies in humans. A systematic review is provided for the development of peptide probes and chelators, as well as a description of labelling with various SPECT and PET tracers including ${ }^{99 \mathrm{~m}} \mathrm{Tc},{ }^{111} \mathrm{In}$, ${ }^{123} \mathrm{I},{ }^{86 / 90} \mathrm{Y},{ }^{177} \mathrm{Lu},{ }^{67 / 68} \mathrm{Ga},{ }^{64 / 67} \mathrm{Cu}$ and ${ }^{18} \mathrm{~F}$.

PET/CT using ${ }^{68} \mathrm{Ga}$ peptides is the second biggest breakthrough since the introduction of PRRI, offering a combination of higher binding affinity, different receptor targeting and superb high-resolution imaging. Decristoforo et.al. [3] 
discuss the status of ${ }^{68} \mathrm{Ge} /{ }^{68} \mathrm{Ga}$ generators, automated radiopharmaceutical preparations and quality parameters. They also highlight the factors influencing the feasibility and availability of this technique in an increasingly demanding regulatory environment worldwide. ${ }^{111}$ In-labelled somatostatin analogues (Octreoscan) remain the most available and used radiopharmaceuticals worldwide, and Pepe et al. [4] describe the standard procedure employed in planar and SPECT imaging. In addition to reviewing the technique, normal findings, variants and clinical applications, they also describe non-octreotide radiopharmaceuticals and provide a brief perspective of future development with the emergence of bivalent and multiagent diagnostic and therapeutic approaches. Despite being the gold standard in PRRI, Octreoscan has been superseded by ${ }^{68} \mathrm{Ga}$-labelled peptide $\mathrm{PET} / \mathrm{CT}$. This significant forward leap offers the advantage of an in-house supply of radiopharmaceutical and outstanding resolution, combined with reduced imaging time and radiation exposure. The next three papers describe the undeniable value of these radiopharmaceuticals. Ambrosini et al. [5] focus on imaging of gastroenteropancreatic NET, which is the most common application of these novel techniques. They describe their experience, and that of others, in the use of ${ }^{68} \mathrm{Ga}$-DOTA-NOC, ${ }^{68} \mathrm{Ga}$-DOTA-TOC, ${ }^{68} \mathrm{Ga}$ DOTA-TATE, and the various other combinations. This is followed by a review of studies comparing ${ }^{68} \mathrm{Ga}$-labelled peptides with other PET tracers in the detection of NET including ${ }^{18} \mathrm{~F}$-DOPA and ${ }^{18} \mathrm{~F}$-FDG.

Naji and AL-Nahhas [6] review the use of ${ }^{68} \mathrm{Ga}$-peptide in neuroectodermal tumours including phaeochromocytomas and paragangliomas. These tumours are usually imaged with ${ }^{123}$ I-MIBG, which has limited resolution and affinity. The authors' experience with ${ }^{68} \mathrm{Ga}$-DOTA-TATE and other clinical and experimental studies with ${ }^{68} \mathrm{Ga}$-DOTA-TOC and ${ }^{68} \mathrm{Ga}$-DOTA-NOC reveals the diagnostic power of these tracers in detecting extra lesions particularly in the skeletal system. Although these tumours are rare, there is disproportionately limited experience in the literature concerning the use of ${ }^{68} \mathrm{Ga}$-based peptides in their management, a fact that requires further and larger studies to establish their full value.

Receptor imaging is not limited to tumours and Roivainen et al. [7] consider the use of ${ }^{68} \mathrm{Ga}$-based peptides in the detection of inflammation. They review the development of labelling of VAP-1, an inflammatory marker, with ${ }^{68} \mathrm{Ga}$-based peptides to form ${ }^{68} \mathrm{Ga}$-DOTAVAP-P1 and its use in a rat model as a proof of concept. Although capable of imaging inflammation, its metabolic half-life is short, necessitating the development of ${ }^{68} \mathrm{Ga}$-DOTAVAP-PEG-P1 with a longer half-life. Glycosylated ArgGly-Asp (RGD) peptides have also been labelled with ${ }^{68} \mathrm{Ga}$; these include ${ }^{68} \mathrm{Ga}$-NODAGA-RGD and ${ }^{68} \mathrm{Ga}$-DOTARGD. Most of these studies are still in the preclinical stage though the results of using ${ }^{68} \mathrm{Ga}$-citrate PET/CT in a clinical setting were satisfactory, despite the short uptake time imposed by the short isotope half-life. Laverman et al. of the Nijmegen group [8] describe receptor targeting with other peptides such as cholecystokinin/gastrin analogues, glucagon-like peptide-1 (GLP-1), bombesin, chemokine receptor CXCR4-targeting peptides, and RGD peptides. These are currently under development or undergoing clinical trials and will further improve the scope of receptor-based diagnosis and therapy.

PRRT was the logical step to follow after the overwhelming success of PRRI. Initial experiences with ${ }^{111}$ In-pentetreotide, using the high-energy, short-range Auger and conversion emissions of ${ }^{111}$ In led to unsatisfactory clinical results. It has now been replaced by two commonly used PRRI radiopharmaceuticals, namely ${ }^{90}$ Y-DOTA-TOC and ${ }^{177}$ Lu-DOTA-TATE. Bodei et al. describe their experience with ${ }^{90}$ Y-DOTA-TOC with overall objective response in $10 \%$ to $34 \%$ of patients [9]. In parallel, Kam et al. of the Rotterdam group review their experience with ${ }^{177}$ Lu-DOTA-TATE and refer to an analysis of 310 treated patients with an overall objective response in $46 \%$ [10]. Both papers describe methods to measure dosimetry and outline the common short-term and long-term toxicity, of which renal toxicity remains of concern. The strategy of coinfusing positively charged amino acids, such as lysine and arginine to competitively inhibit reabsorption of labelled peptides has lead to a substantial reduction in the renal doses, but opinions differ as to which amino acids to use and for how long. This also applies to treatment methodology whereby all published results have been derived from different inhomogeneous phase I/II studies. Presently, PRRT protocols rely mostly on empirical criteria using standard activities, as derived from escalation studies, clinical experience, or historical reasons. There are huge differences among protocols, as to activities, fixed or related to body weight or surface, number of cycles, and time intervals between cycles. Likewise, the optimal timing of PRRI in the management of SSR-positive tumours and the way in which it should be integrated with other treatment have yet to be defined. Protocols combining ${ }^{177} \mathrm{Lu}$ - and ${ }^{90}$ Y-peptides have been recently contemplated, in order to benefit from the different physical properties of the radionuclides, though activities of ${ }^{177} \mathrm{Lu}$ and ${ }^{90} \mathrm{Y}$ have not been based on dosimetric values. It is very clear that prospective phase II/III trials comparing the efficacy and toxicity of different schemes of ${ }^{90}$ Y-DOTA-TOC administration are still warranted.

To cover the entire spectrum of therapy, Bomanji and Papathanasiou [11] review the literature on NET radionuclide therapy with ${ }^{111}$ In-DTPA ${ }^{0}$-octreotide and ${ }^{131}$ I-MIBG, 
focusing on efficacy and toxicity. ${ }^{131}$ I-MIBG is the radionuclide therapy of choice for inoperable or metastatic phaeochromocytomas/paragangliomas with symptomatic, biochemical and morphological responses of $50-89 \%, 45-$ $74 \%$ and $27-47 \%$ respectively. Other radiopharmaceuticals such meta-astato $\left({ }^{211} \mathrm{At}\right)$-benzylguanidine (MABG) and radiolabelled exendin await integration into clinical practice.

Finally, the role of RGD peptides in diagnosis and therapy is discussed by Gaertner et al. [12]. They explain the reasons behind the choice of molecular markers for the assessment of angiogenic activity of tumours. The integrin-specific radiotracers are obtained by labelling RGD-based compounds such as Integrin $\alpha_{\mathrm{v}} \beta_{3}$, which is highly expressed on the cell surface of activated endothelial cells in newly formed blood vessels. Further data on the ultimate clinical value of imaging of integrin expression are still awaited.

We hope that this supplement will serve as a historical review of the role of peptides in diagnosis and therapy, an update of the present state-of-the-art techniques and availability as well as a predictor of what the future holds for this exciting chapter in nuclear medicine. The successful imaging of NET and other tumours using receptor-based PET/CT radiopharmaceuticals will hopefully increase the demand for them and subsequently improve their widespread availability.

We appreciate that the reader may notice some overlap, occasional repetition and minor contradictions based on personal expertise and local availability. This is inevitable; particularly where PRRT is concerned, and may only help to further the efforts to conduct larger multicentre studies to find the best way forward.

Conflicts of interest None.

\section{References}

1. Koopmans KP, Glaudemans AW. Rationale for the use of radiolabelled peptides in diagnosis and therapy. Eur J Nucl Med Mol Imaging. doi:10.1007/s00259-011-2038-Z

2. Fani M, Maecke HR. Radiopharmaceutical development of radiolabelled peptides. Eur J Nucl Med Mol Imaging. doi:10.1007/ s00259-011-2001-z

3. Decristoforo C, Pickett RD, Verbruggen A. Feasibility and availability of $68 \mathrm{Ga}-$ labelled peptides. Eur J Nucl Med Mol Imaging. doi:10.1007/s00259-011-1988-5

4. Pepe G, Moncayo R, Bombardieri E, Chiti A. Somatostatin receptor SPECT. Eur J Nucl Med Mol Imaging. doi:10.1007/s00259011-2019-2

5. Ambrosini V, Campana D, Tomassetti P, Fanti S. 68Ga-labelled peptides for diagnosis of gastroenteropancreatic NET. Eur J Nucl Med Mol Imaging. doi:10.1007/s00259-011-1989-4

6. Naji M, AL-Nahhas A. $68 \mathrm{Ga}$-labelled peptides in the management of neuroectodermal tumours. Eur J Nucl Med Mol Imaging. doi:10.1007/s00259-011-1990-y

7. Roivainen A, Jalkanen S, Nanni C. Gallium-labelled peptides for imaging of inflammation. Eur J Nucl Med Mol Imaging. doi:10.1007/s00259-011-1987-6

8. Laverman P, Sosabowski JK, Boerman OC, Oyen WJ. Radiolabelled peptides for oncological diagnosis. Eur J Nucl Med Mol Imaging. doi:10.1007/s00259-011-2014-7

9. Bodei L, Cremonesi M, Grana CM, Chinol M, Baio SM, Severi S, et al. Yttrium-labelled peptides for therapy of NET. Eur J Nucl Med Mol Imaging. doi:10.1007/s00259-011-2002-y

10. Kam BL, Teunissen JJ, Krenning EP, de Herder WW, Khan S, van Vliet EI, et al. Lutetium-labelled peptides for therapy of neuroendocrine tumours. Eur J Nucl Med Mol Imaging. doi:10.1007/ s00259-011-2039-y

11. Bomanji JB, Papathanasiou ND. 111 In-DTPA0-octreotide (Octreoscan), 131I-MIBG and other agents for radionuclide therapy of NETs. Eur J Nucl Med Mol Imaging. doi:10.1007/s00259-011-2013-8

12. Gaertner FC, Kessler H, Wester H.-J, Schwaiger M, Beer AJ. Radiolabelled RGD peptides for imaging and therapy. Eur J Nucl Med Mol Imaging. doi:10.1007/s00259-011-2028-1 